\title{
Convergence of Iterative Sequences for Generalized Equilibrium Problems Involving Inverse-Strongly Monotone Mappings
}

\author{
Shin Min Kang, ${ }^{1}$ Sun Young Cho, ${ }^{2}$ and Zeqing Liu ${ }^{3}$ \\ ${ }^{1}$ Department of Mathematics and the RINS, Gyeongsang National University, Jinju 660-701, South Korea \\ ${ }^{2}$ Department of Mathematics, Gyeongsang National University, Jinju 660-701, South Korea \\ ${ }^{3}$ Department of Mathematics, Liaoning Normal University, Dalian, Liaoning 116029, China \\ Correspondence should be addressed to Sun Young Cho, ooly61@yahoo.co.kr \\ Received 11 December 2009; Accepted 25 January 2010 \\ Academic Editor: Yeol Je Cho \\ Copyright (C) 2010 Shin Min Kang et al. This is an open access article distributed under the Creative \\ Commons Attribution License, which permits unrestricted use, distribution, and reproduction in \\ any medium, provided the original work is properly cited. \\ The purpose of this paper is to consider the weak convergence of an iterative sequence for finding a \\ common element in the set of solutions of generalized equilibrium problems, in the set of solutions \\ of classical variational inequalities, and in the set of fixed points of nonexpansive mappings.
}

\section{Introduction and Preliminaries}

Throughout this paper, we always assume that $H$ is a real Hilbert space with the inner product $\langle\cdot, \cdot \cdot\rangle$ and the norm $\|\cdot\|$ and $C$ is a nonempty closed convex subset of $H$. Let $S: C \rightarrow C$ be a nonlinear mapping. In this paper, we use $F(S)$ to denote the fixed point set of $S$. Recall that the mapping $S$ is said to be nonexpansive if

$$
\|S x-S y\| \leq\|x-y\|, \quad \forall x, y \in C .
$$

Let $A: C \rightarrow H$ be a mapping. Recall that $A$ is said to be monotone if

$$
\langle A x-A y, x-y\rangle \geq 0, \quad \forall x, y \in C .
$$


$A$ is said to be inverse-strongly monotone if there exists a constant $\alpha>0$ such that

$$
\langle A x-A y, x-y\rangle \geq \alpha\|A x-A y\|^{2}, \quad \forall x, y \in C .
$$

A set-valued mapping $R: H \rightarrow 2^{H}$ is said to be monotone if for all $x, y \in H, f \in R x$ and $g \in R y$ imply $\langle x-y, f-g\rangle>0$. A monotone mapping $R: H \rightarrow 2^{H}$ is maximal if the graph $G(R)$ of $R$ is not properly contained in the graph of any other monotone mapping. It is known that a monotone mapping $R$ is maximal if and only if, for any $(x, f) \in H \times H,\langle x-y, f-g\rangle \geq 0$ for all $(y, g) \in G(R)$ implies $f \in R x$.

Let $F$ be a bifunction of $C \times C$ into $\mathbb{R}$, where $\mathbb{R}$ denotes the set of real numbers and $A: C \rightarrow H$ an inverse-strongly monotone mapping. In this paper, we consider the following generalized equilibrium problem:

$$
\text { Find } x \in C \text { such that } F(x, y)+\langle A x, y-x\rangle \geq 0, \quad \forall y \in C \text {. }
$$

In this paper, the set of such an $x \in C$ is denoted by $\operatorname{EP}(F, A)$, that is,

$$
\mathrm{EP}(F, A)=\{x \in C: F(x, y)+\langle A x, y-x\rangle \geq 0, \forall y \in C\}
$$

Next, we give two special cases of problem (1.4).

(I) If $A \equiv 0$, then the generalized equilibrium problem (1.4) is reduced to the following equilibrium problem:

$$
\text { Find } x \in C \text { such that } F(x, y) \geq 0, \quad \forall y \in C \text {. }
$$

In this paper, the set of such an $x \in C$ is denoted by $\mathrm{EP}(F)$, that is,

$$
\mathrm{EP}(F)=\{x \in C: F(x, y) \geq 0, \forall y \in C\}
$$

Numerous problems in physics, optimization, and economics reduce to finding a solution of the equilibrium problem. conditions:

To study problems (1.4) and (1.6), we may assume that $F$ satisfies the following

(A1) $F(x, x)=0$ for all $x \in C$;

(A2) $F$ is monotone, that is, $F(x, y)+F(y, x) \leq 0$ for all $x, y \in C$;

(A3) for each $x, y, z \in C$,

$$
\limsup _{t \downarrow 0} F(t z+(1-t) x, y) \leq F(x, y)
$$

(A4) for each $x \in C, y \mapsto F(x, y)$ is convex and weakly lower semicontinuous. 
(II) If $F \equiv 0$, then problem (1.4) is reduced to the classical variational inequality. Find $x \in C$ such that

$$
\langle A x, y-x\rangle \geq 0, \quad \forall y \in C
$$

It is known that $x \in C$ is a solution to (1.9) if and only if $x$ is a fixed point of the mapping $P_{C}(I-\lambda A)$, where $\lambda>0$ is a constant and $I$ is the identity mapping.

In 2003, Takahashi and Toyoda [1] considered the variational inequality (1.9) and proved the following theorem.

Theorem 1.1. Let $C$ be a closed convex subset of a real Hilbert space $H$. Let $A$ be an $\alpha$-inversestrongly monotone mapping of $C$ into $H$, and let $S$ be a nonexpansive mapping of $C$ into itself such that $\mathcal{F}=F(S) \cap \operatorname{VI}(C, A) \neq \emptyset$. Let $\left\{x_{n}\right\}$ be a sequence generated by

$$
x_{0} \in C, \quad x_{n+1}=\alpha_{n} x_{n}+\left(1-\alpha_{n}\right) S P_{C}\left(x_{n}-\lambda_{n} A x_{n}\right), \quad \forall n \geq 0,
$$

where $\lambda_{n} \in[a, b]$ for some $a, b \in(0,2 \alpha)$ and $\alpha_{n} \in[c, d]$ for some $c, d \in(0,1)$. Then, $\left\{x_{n}\right\}$ converges weakly to $z \in F(S) \cap V I(C, A)$, where $z=\lim _{n \rightarrow \infty} P_{\mp} x_{n}$.

In 2007, Tada and Takahashi [2] considered the equilibrium problem (1.6) and proved the following result.

Theorem 1.2. Let $C$ be a nonempty closed convex subset of $H$. Let $F$ be a bifunction from $C \times C$ to $\mathbb{R}$ satisfying (A1)-(A4) and let $S$ be a nonexpansive mapping of $C$ into $H$ such that $F(S) \cap \operatorname{EP}(F) \neq \emptyset$. Let $\left\{x_{n}\right\}$ and $\left\{u_{n}\right\}$ be sequences generated by $x_{1}=x \in H$ and let

$$
\begin{gathered}
u_{n} \in C \text { such that } F\left(u_{n}, u\right)+\frac{1}{r_{n}}\left\langle u-u_{n}, u_{n}-x_{n}\right\rangle \geq 0, \quad \forall u \in C, \\
x_{n+1}=\alpha_{n} x_{n}+\left(1-\alpha_{n}\right) S u_{n}, \quad \forall n \geq 1,
\end{gathered}
$$

where $\left\{\alpha_{n}\right\} \subset[a, b]$ for some $a, b \in(0,1)$ and $\left\{r_{n}\right\} \subset(0, \infty)$ satisfies $\liminf _{n \rightarrow \infty} r_{n}>0$. Then, $\left\{x_{n}\right\}$ converges weakly to $w \in F(S) \cap \mathrm{EP}(F)$, where $w=\lim _{n \rightarrow \infty} P_{F(S) \cap \mathrm{EP}(F)} x_{n}$.

Very recently, Moudafi [3] considered the following iterative process:

$$
\begin{gathered}
x_{0} \in C, \\
F_{1}\left(u_{n}, u\right)+\frac{1}{r_{n}}\left\langle u-u_{n}, u_{n}-x_{n}\right\rangle \geq 0, \quad \forall u \in C, \\
F_{2}\left(v_{n}, v\right)+\frac{1}{r_{n}}\left\langle v-v_{n}, v_{n}-x_{n}\right\rangle \geq 0, \quad \forall v \in C, \\
x_{n+1}=\frac{u_{n}+v_{n}}{2}, \quad \forall n \geq 1,
\end{gathered}
$$

where $F_{1}$ and $F_{2}$ are bifunctions and $\left\{r_{n}\right\}$ is a control sequence. A weak convergence theorem was established; see [3] for more details. 
Weak convergence of iterative sequences has been studied recently for the problems (1.4), (1.6), and (1.9); see [1-14] and the references therein. In this paper, we consider the generalized equilibrium problem (1.4) and a nonexpansive mapping based on an iterative process. We show that the sequence generated in the purposed iterative process converges weakly to a common element in the set of solutions of the variational inequality (1.9), in the fixed point sets of a nonexpansive mapping and in the solution sets of the generalized equilibrium problem (1.4). The results presented in this paper improve and extend the corresponding results announced by Takahashi and Toyoda [1] and Tada and Takahashi [2].

In order to prove our main results, we also need the following lemmas.

Lemma 1.3 (see [1]). Let $C$ be a nonempty closed convex subset of $H$. Let $\left\{x_{n}\right\}$ be a sequence in $H$. Suppose that, for all $y \in C$,

$$
\left\|x_{n+1}-y\right\| \leq\left\|x_{n}-y\right\|, \quad \forall n \geq 1
$$

then $\left\{P_{C}\left(x_{n}\right)\right\}$ converges strongly to some $z \in C$.

The following lemma can be found in [15].

Lemma 1.4. Let $T$ be a monotone mapping of $C$ into $H$ and $N_{C} v$ the normal cone to $C$ at $v \in C$, that is,

$$
N_{C} v=\{w \in H:\langle v-u, w\rangle \geq 0, \forall u \in C\}
$$

and define a mapping $R$ on $C$ by

$$
R v= \begin{cases}T v+N_{C} v, & v \in C, \\ \emptyset, & v \notin C .\end{cases}
$$

Then $R$ is maximal monotone and $0 \in R v$ if and only if $\langle T v, u-v\rangle \geq 0$ for all $u \in C$.

The following lemma can be found in $[16,17]$.

Lemma 1.5. Let $C$ be a nonempty closed convex subset of $H$ and let $F: C \times C \rightarrow \mathbb{R}$ be a bifunction satisfying (A1)-(A4). Then, for any $r>0$ and $x \in H$, there exists $z \in C$ such that

$$
F(z, y)+\frac{1}{r}\langle y-z, z-x\rangle \geq 0, \quad \forall y \in C
$$

Further, define

$$
T_{r} x=\left\{z \in C: F(z, y)+\frac{1}{r}\langle y-z, z-x\rangle \geq 0, \forall y \in C\right\}
$$


for all $r>0$ and $x \in H$. Then, the following hold:

(a) $T_{r}$ is single-valued;

(b) $T_{r}$ is firmly nonexpansive, that is, for any $x, y \in H$,

$$
\left\|T_{r} x-T_{r} y\right\|^{2} \leq\left\langle T_{r} x-T_{r} y, x-y\right\rangle
$$

(c) $F\left(T_{r}\right)=\mathrm{EP}(F)$;

(d) $\mathrm{EP}(F)$ is closed and convex.

Lemma 1.6 (see [18]). Let $H$ be a Hilbert space and $0<p \leq t_{n} \leq q<1$ for all $n \geq 1$. Suppose that $\left\{x_{n}\right\}$ and $\left\{y_{n}\right\}$ are sequences in $H$ such that

$$
\begin{gathered}
\limsup _{n \rightarrow \infty}\left\|x_{n}\right\| \leq r, \quad \limsup _{n \rightarrow \infty}\left\|y_{n}\right\| \leq r, \\
\lim _{n \rightarrow \infty}\left\|t_{n} x_{n}+\left(1-t_{n}\right) y_{n}\right\|=r
\end{gathered}
$$

hold for some $r \geq 0$, then $\lim _{n \rightarrow \infty}\left\|x_{n}-y_{n}\right\|=0$.

Lemma 1.7 (see [19]). Let $H$ be a real Hilbert space, $C$ a nonempty closed convex subset of $H$, and $S: C \rightarrow C$ a nonexpansive mapping. Then the mapping $I-S$ is demiclosed at zero, that is, if $\left\{x_{n}\right\}$ is a sequence in $C$ such that $x_{n} \rightarrow \bar{x}$ and $x_{n}-S x_{n} \rightarrow 0$, then $\bar{x} \in F(S)$.

\section{Main Results}

Theorem 2.1. Let $C$ be a nonempty closed convex subset of a real Hilbert space $H$. Let $F_{1}$ and $F_{2}$ be two bifunctions from $C \times C$ to $\mathbb{R}$ which satisfy (A1)-(A4). Let $A: C \rightarrow H$ be an $\alpha$-inversestrongly monotone mapping, $B: C \rightarrow H$ a $\beta$-inverse-strongly monotone mapping, $T: C \rightarrow H$ an $\lambda$-inverse-strongly monotone mapping, and $S: C \rightarrow C$ a nonexpansive mapping. Assume that $\mathcal{F}:=\operatorname{EP}\left(F_{1}, A\right) \cap \operatorname{EP}\left(F_{2}, B\right) \cap V I(C, T) \cap F(S) \neq \emptyset$. Let $\left\{\alpha_{n}\right\}$ and $\left\{\beta_{n}\right\}$ be sequences in $[0,1]$. Let $\left\{a_{n}\right\}$ be a sequence in $[0,2 \alpha],\left\{b_{n}\right\}$ a sequence in $[0,2 \beta]$, and $\left\{t_{n}\right\}$ a sequence in $[0,2 \lambda]$. Let $\left\{x_{n}\right\}$ be a sequence generated in the following manner:

$$
\begin{gathered}
x_{1} \in C, \\
F_{1}\left(u_{n}, u\right)+\left\langle A x_{n}, u-u_{n}\right\rangle+\frac{1}{a_{n}}\left\langle u-u_{n}, u_{n}-x_{n}\right\rangle \geq 0, \quad \forall u \in C, \\
F_{2}\left(v_{n}, v\right)+\left\langle B x_{n}, v-v_{n}\right\rangle+\frac{1}{b_{n}}\left\langle v-v_{n}, v_{n}-x_{n}\right\rangle \geq 0, \quad \forall v \in C, \\
y_{n}=\beta_{n} u_{n}+\left(1-\beta_{n}\right) v_{n}, \\
x_{n+1}=\alpha_{n} x_{n}+\left(1-\alpha_{n}\right) S P_{C}\left(y_{n}-t_{n} T y_{n}\right), \quad \forall n \geq 1 .
\end{gathered}
$$


Assume that the sequences $\left\{\alpha_{n}\right\},\left\{\beta_{n}\right\},\left\{a_{n}\right\},\left\{b_{n}\right\}$, and $\left\{t_{n}\right\}$ satisfy the following restrictions:

(a) $0<a^{\prime} \leq \alpha_{n} \leq a<1,0<b \leq \beta_{n} \leq c<1$;

(b) $0<d \leq a_{n} \leq e<2 \alpha, 0<f \leq b_{n} \leq g<2 \beta, 0<h \leq t_{n} \leq j<2 \lambda$

for some $a^{\prime}, a, b, c, d, e, f, g, h, j \in \mathbb{R}$, then the sequence $\left\{x_{n}\right\}$ generated in $(\Delta)$ converges weakly to some point $\bar{x} \in \mathcal{F}$, where $\bar{x}=\lim _{n \rightarrow \infty} P_{\bar{F}} x_{n}$.

Proof. Fix $p \in \mathcal{F}$. It follows that

$$
p=S p=T_{a_{n}}\left(I-a_{n} A\right) p=T_{b_{n}}\left(I-b_{n} B\right) p=P_{C}\left(I-t_{n} T\right) p, \quad \forall n \geq 1 .
$$

Note that $I-t_{n} T$ is nonexpansive for each $n \geq 1$. Indeed, for any $x, y \in C$, we see that

$$
\begin{aligned}
\left\|\left(I-t_{n} T\right) x-\left(I-t_{n} T\right) y\right\|^{2} & =\left\|(x-y)-t_{n}(T x-T y)\right\|^{2} \\
& =\|x-y\|^{2}-2 t_{n}\langle x-y, T x-T y\rangle+t_{n}^{2}\|T x-T y\|^{2} \\
& \leq\|x-y\|^{2}-t_{n}\left(2 \lambda-t_{n}\right)\|T x-T y\|^{2} \\
& \leq\|x-y\|^{2} .
\end{aligned}
$$

In a similar way, we can obtain that $I-a_{n} A$ and $I-b_{n} B$ are nonexpansive for each $n \geq 1$. Note that

$$
\begin{gathered}
\left\|u_{n}-p\right\| \leq\left\|T_{a_{n}}\left(I-a_{n} A\right) x_{n}-p\right\| \leq\left\|x_{n}-p\right\|, \\
\left\|v_{n}-p\right\| \leq\left\|T_{b_{n}}\left(I-b_{n} B\right) x_{n}-p\right\| \leq\left\|x_{n}-p\right\| .
\end{gathered}
$$

Put $z_{n}=P_{C}\left(y_{n}-t_{n} T y_{n}\right)$ for each $n \geq 1$. It follows from (2.3) that

$$
\begin{aligned}
\left\|x_{n+1}-p\right\| & \leq \alpha_{n}\left\|x_{n}-p\right\|+\left(1-\alpha_{n}\right)\left\|S z_{n}-p\right\| \\
& \leq \alpha_{n}\left\|x_{n}-p\right\|+\left(1-\alpha_{n}\right)\left\|z_{n}-p\right\| \\
& \leq \alpha_{n}\left\|x_{n}-p\right\|+\left(1-\alpha_{n}\right)\left\|y_{n}-p\right\| \\
& \leq \alpha_{n}\left\|x_{n}-p\right\|+\left(1-\alpha_{n}\right)\left(\beta_{n}\left\|u_{n}-p\right\|+\left(1-\beta_{n}\right)\left\|v_{n}-p\right\|\right) \\
& \leq\left\|x_{n}-p\right\| .
\end{aligned}
$$

This implies that $\lim _{n \rightarrow \infty}\left\|x_{n}-p\right\|$ exists. This shows that $\left\{x_{n}\right\}$ is bounded, so are $\left\{y_{n}\right\},\left\{u_{n}\right\}$, and $\left\{v_{n}\right\}$.

On the other hand, we have

$$
\begin{gathered}
\left\|u_{n}-p\right\|^{2}=\left\|T_{a_{n}}\left(I-a_{n} A\right) x_{n}-p\right\|^{2} \leq\left\|x_{n}-p\right\|^{2}-a_{n}\left(2 \alpha-a_{n}\right)\left\|A x_{n}-A p\right\|^{2}, \\
\left\|v_{n}-p\right\|^{2}=\left\|T_{b_{n}}\left(I-b_{n} B\right) x_{n}-p\right\|^{2} \leq\left\|x_{n}-p\right\|^{2}-b_{n}\left(2 \beta-b_{n}\right)\left\|B x_{n}-B p\right\|^{2} .
\end{gathered}
$$


Combining (2.5) with (2.6) yields that

$$
\begin{aligned}
\left\|x_{n+1}-p\right\|^{2} \leq & \alpha_{n}\left\|x_{n}-p\right\|^{2}+\left(1-\alpha_{n}\right)\left\|S z_{n}-p\right\|^{2} \\
& \leq \alpha_{n}\left\|x_{n}-p\right\|^{2}+\left(1-\alpha_{n}\right)\left\|z_{n}-p\right\|^{2} \\
& \leq \alpha_{n}\left\|x_{n}-p\right\|^{2}+\left(1-\alpha_{n}\right)\left\|y_{n}-p\right\|^{2} \\
& \leq \alpha_{n}\left\|x_{n}-p\right\|^{2}+\left(1-\alpha_{n}\right)\left(\beta_{n}\left\|u_{n}-p\right\|^{2}+\left(1-\beta_{n}\right)\left\|v_{n}-p\right\|^{2}\right) \\
& \leq \alpha_{n}\left\|x_{n}-p\right\|^{2}+\left(1-\alpha_{n}\right)\left(\beta_{n}\left(\left\|x_{n}-p\right\|^{2}-a_{n}\left(2 \alpha-a_{n}\right)\left\|A x_{n}-A p\right\|^{2}\right)\right. \\
& \left.\quad+\left(1-\beta_{n}\right)\left(\left\|x_{n}-p\right\|^{2}-b_{n}\left(2 \beta-a_{n}\right)\left\|B x_{n}-B p\right\|^{2}\right)\right) \\
& \leq\left\|x_{n}-p\right\|^{2}-\left(1-\alpha_{n}\right) \beta_{n} a_{n}\left(2 \alpha-a_{n}\right)\left\|A x_{n}-A p\right\|^{2} \\
& \quad-\left(1-\alpha_{n}\right)\left(1-\beta_{n}\right) b_{n}\left(2 \beta-a_{n}\right)\left\|B x_{n}-B p\right\|^{2} .
\end{aligned}
$$

This implies that

$$
\left(1-\alpha_{n}\right) \beta_{n} a_{n}\left(2 \alpha-a_{n}\right)\left\|A x_{n}-A p\right\|^{2} \leq\left\|x_{n}-p\right\|^{2}-\left\|x_{n+1}-p\right\|^{2} .
$$

In view of the restrictions (a) and (b), we obtain that

$$
\lim _{n \rightarrow \infty}\left\|A x_{n}-A p\right\|=0
$$

It also follows from (2.7) that

$$
\left(1-\alpha_{n}\right)\left(1-\beta_{n}\right) b_{n}\left(2 \beta-a_{n}\right)\left\|B x_{n}-B p\right\|^{2} \leq\left\|x_{n}-p\right\|^{2}-\left\|x_{n+1}-p\right\|^{2} .
$$

In view of the restrictions (a) and (b), we obtain that

$$
\lim _{n \rightarrow \infty}\left\|B x_{n}-B p\right\|=0
$$


On the other hand, we see from Lemma 1.5 that

$$
\begin{aligned}
\left\|u_{n}-p\right\|^{2}= & \left\|T_{a_{n}}\left(I-a_{n} A\right) x_{n}-T_{a_{n}}\left(I-a_{n} A\right) p\right\|^{2} \\
\leq & \left\langle\left(I-a_{n} A\right) x_{n}-\left(I-a_{n} A\right) p, u_{n}-p\right\rangle \\
= & \frac{1}{2}\left(\left\|\left(I-a_{n} A\right) x_{n}-\left(I-a_{n} A\right) p\right\|^{2}+\left\|u_{n}-p\right\|^{2}\right. \\
& \left.\quad-\left\|\left(I-a_{n} A\right) x_{n}-\left(I-a_{n} A\right) p-\left(u_{n}-p\right)\right\|^{2}\right) \\
\leq & \frac{1}{2}\left(\left\|x_{n}-p\right\|^{2}+\left\|u_{n}-p\right\|^{2}-\left\|x_{n}-u_{n}-a_{n}\left(A x_{n}-A p\right)\right\|^{2}\right) \\
= & \frac{1}{2}\left(\left\|x_{n}-p\right\|^{2}+\left\|u_{n}-p\right\|^{2}\right. \\
& \left.\quad-\left(\left\|x_{n}-u_{n}\right\|^{2}-2 a_{n}\left\langle x_{n}-u_{n}, A x_{n}-A p\right\rangle+a_{n}^{2}\left\|A x_{n}-A p\right\|^{2}\right)\right) .
\end{aligned}
$$

This implies that

$$
\left\|u_{n}-p\right\|^{2} \leq\left\|x_{n}-p\right\|^{2}-\left\|x_{n}-u_{n}\right\|^{2}+2 a_{n}\left\|x_{n}-u_{n}\right\|\left\|A x_{n}-A p\right\| .
$$

In a similar way, we can obtain that

$$
\left\|v_{n}-p\right\|^{2} \leq\left\|x_{n}-p\right\|^{2}-\left\|x_{n}-v_{n}\right\|^{2}+2 b_{n}\left\|x_{n}-v_{n}\right\|\left\|B x_{n}-B p\right\| .
$$

It follows from (2.13) and (2.14) that

$$
\begin{aligned}
\left\|x_{n+1}-p\right\|^{2} \leq & \alpha_{n}\left\|x_{n}-p\right\|^{2}+\left(1-\alpha_{n}\right)\left\|S z_{n}-p\right\|^{2} \\
\leq & \alpha_{n}\left\|x_{n}-p\right\|^{2}+\left(1-\alpha_{n}\right)\left\|z_{n}-p\right\|^{2} \\
\leq & \alpha_{n}\left\|x_{n}-p\right\|^{2}+\left(1-\alpha_{n}\right)\left\|y_{n}-p\right\|^{2} \\
\leq & \alpha_{n}\left\|x_{n}-p\right\|^{2}+\left(1-\alpha_{n}\right)\left(\beta_{n}\left\|u_{n}-p\right\|^{2}+\left(1-\beta_{n}\right)\left\|v_{n}-p\right\|^{2}\right) \\
\leq & \alpha_{n}\left\|x_{n}-p\right\|^{2}+\left(1-\alpha_{n}\right) \\
& \times\left(\beta_{n}\left(\left\|x_{n}-p\right\|^{2}-\left\|x_{n}-u_{n}\right\|^{2}+2 a_{n}\left\|x_{n}-u_{n}\right\|\left\|A x_{n}-A p\right\|\right)\right. \\
& \left.\quad+\left(1-\beta_{n}\right)\left(\left\|x_{n}-p\right\|^{2}-\left\|x_{n}-v_{n}\right\|^{2}+2 b_{n}\left\|x_{n}-v_{n}\right\|\left\|B x_{n}-B p\right\|\right)\right) \\
\leq & \left\|x_{n}-p\right\|^{2}-\left(1-\alpha_{n}\right) \beta_{n}\left\|x_{n}-u_{n}\right\|^{2}+2 a_{n}\left\|x_{n}-u_{n}\right\|\left\|A x_{n}-A p\right\| \\
& \quad\left(1-\alpha_{n}\right)\left(1-\beta_{n}\right)\left\|x_{n}-v_{n}\right\|^{2}+2 b_{n}\left\|x_{n}-v_{n}\right\|\left\|B x_{n}-B p\right\| .
\end{aligned}
$$


This shows that

$$
\begin{aligned}
\left(1-\alpha_{n}\right) \beta_{n}\left\|x_{n}-u_{n}\right\|^{2} \leq & \left\|x_{n}-p\right\|^{2}-\left\|x_{n+1}-p\right\|^{2}+2 a_{n}\left\|x_{n}-u_{n}\right\|\left\|A x_{n}-A p\right\| \\
& +2 b_{n}\left\|x_{n}-v_{n}\right\|\left\|B x_{n}-B p\right\| .
\end{aligned}
$$

In view of the restriction (a), we obtain from (2.9) and (2.11) that

$$
\lim _{n \rightarrow \infty}\left\|x_{n}-u_{n}\right\|=0
$$

From (2.15), we also have

$$
\begin{aligned}
\left(1-\alpha_{n}\right)\left(1-\beta_{n}\right)\left\|x_{n}-v_{n}\right\|^{2} \leq & \left\|x_{n}-p\right\|^{2}-\left\|x_{n+1}-p\right\|^{2}+2 a_{n}\left\|x_{n}-u_{n}\right\|\left\|A x_{n}-A p\right\| \\
& +2 b_{n}\left\|x_{n}-v_{n}\right\|\left\|B x_{n}-B p\right\| .
\end{aligned}
$$

In view of the restriction (a), we obtain from (2.9) and (2.11) that

$$
\lim _{n \rightarrow \infty}\left\|x_{n}-v_{n}\right\|=0
$$

Since $\left\{x_{n}\right\}$ is bounded, we see that there exits a subsequence $\left\{x_{n_{i}}\right\}$ of $\left\{x_{n}\right\}$ which converges weakly to $\bar{x}$. It follows from (2.17) that $u_{n_{i}}$ converges weakly to $\bar{x}$. Note that

$$
F_{1}\left(u_{n}, u\right)+\left\langle A x_{n}, u-u_{n}\right\rangle+\frac{1}{a_{n}}\left\langle u-u_{n}, u_{n}-x_{n}\right\rangle \geq 0, \quad \forall u \in C
$$

From (A2), we see that

$$
\left\langle A x_{n}, u-u_{n}\right\rangle+\frac{1}{a_{n}}\left\langle u-u_{n}, u_{n}-x_{n}\right\rangle \geq F_{1}\left(u, u_{n}\right), \quad \forall u \in C .
$$

Replacing $n$ by $n_{i}$, we arrive at

$$
\left\langle A x_{n_{i}}, u-u_{n_{i}}\right\rangle+\frac{1}{a_{n_{i}}}\left\langle u-u_{n_{i}}, u_{n_{i}}-x_{n_{i}}\right\rangle \geq F_{1}\left(u, u_{n_{i}}\right), \quad \forall u \in C .
$$

For $t$ with $0<t \leq 1$ and $u \in C$, let $u_{t}=t u+(1-t) \bar{x}$. Since $u \in C$ and $\bar{x} \in C$, we have $u_{t} \in C$. It follows from (2.22) that

$$
\begin{aligned}
\left\langle u_{t}-u_{n_{i}}, A u_{t}\right\rangle \geq & \left\langle u_{t}-u_{n_{i}}, A u_{t}\right\rangle-\left\langle A x_{n_{i}}, u_{t}-u_{n_{i}}\right\rangle-\left\langle u_{t}-u_{n_{i}}, \frac{u_{n_{i}}-x_{n_{i}}}{a_{n_{i}}}\right\rangle+F_{1}\left(u_{t}, u_{n_{i}}\right) \\
= & \left\langle u_{t}-u_{n_{i}}, A u_{t}-A u_{n_{i}}\right\rangle+\left\langle u_{t}-u_{n_{i}}, A u_{n_{i}}-A x_{n_{i}}\right\rangle \\
& -\left\langle u_{t}-u_{n_{i}}, \frac{u_{n_{i}}-x_{n_{i}}}{a_{n_{i}}}\right\rangle+F_{1}\left(u_{t}, u_{n_{i}}\right) .
\end{aligned}
$$


From (2.17), we have $A u_{n_{i}}-A x_{n_{i}} \rightarrow 0$ as $i \rightarrow \infty$. On the other hand, we obtain from the monotonicity of $A$ that $\left\langle u_{t}-u_{n_{i}}, A u_{t}-A u_{n_{i}}\right\rangle \geq 0$. It follows from (A4) that

$$
\left\langle u_{t}-\bar{x}, A u_{t}\right\rangle \geq F_{1}\left(u_{t}, \bar{x}\right)
$$

From (A1), (A4), and (2.24), we obtain that

$$
\begin{aligned}
0 & =F_{1}\left(u_{t}, u_{t}\right) \leq t F_{1}\left(u_{t}, u\right)+(1-t) F_{1}\left(u_{t}, \bar{x}\right) \\
& \leq t F_{1}\left(u_{t}, u\right)+(1-t)\left\langle u_{t}-\bar{x}, A u_{t}\right\rangle \\
& =t F_{1}\left(u_{t}, u\right)+(1-t) t\left\langle u-\bar{x}, A u_{t}\right\rangle,
\end{aligned}
$$

which yields that

$$
F_{1}\left(u_{t}, u\right)+(1-t)\left\langle u-\bar{x}, A u_{t}\right\rangle \geq 0
$$

Letting $t \rightarrow 0$ in the above inequality, we arrive at

$$
F_{1}(\bar{x}, u)+\langle u-\bar{x}, A \bar{x}\rangle \geq 0
$$

This shows that $\bar{x} \in \operatorname{EP}\left(F_{1}, A\right)$. In a similar way, we can obtain that $\bar{x} \in \operatorname{EP}\left(F_{2}, B\right)$.

Next, we claim that $\bar{x} \in V I(C, T)$

$$
\begin{aligned}
\left\|x_{n+1}-p\right\|^{2} & \leq \alpha_{n}\left\|x_{n}-p\right\|^{2}+\left(1-\alpha_{n}\right)\left\|S P_{C}\left(y_{n}-t_{n} T y_{n}\right)-p\right\|^{2} \\
& \leq \alpha_{n}\left\|x_{n}-p\right\|^{2}+\left(1-\alpha_{n}\right)\left\|\left(y_{n}-t_{n} T y_{n}\right)-\left(p-t_{n} T p\right)\right\|^{2} \\
& \leq \alpha_{n}\left\|x_{n}-p\right\|^{2}+\left(1-\alpha_{n}\right)\left(\left\|y_{n}-p\right\|^{2}-t_{n}\left(2 \lambda-t_{n}\right)\left\|T y_{n}-T p\right\|^{2}\right) \\
& \leq\left\|x_{n}-p\right\|^{2}-\left(1-\alpha_{n}\right) t_{n}\left(2 \lambda-t_{n}\right)\left\|T \mathrm{y}_{n}-T p\right\|^{2} .
\end{aligned}
$$

It follows that

$$
\left(1-\alpha_{n}\right) t_{n}\left(2 \lambda-t_{n}\right)\left\|T y_{n}-T p\right\|^{2} \leq\left\|x_{n}-p\right\|^{2}-\left\|x_{n+1}-p\right\|^{2} .
$$

This implies from conditions (a) and (b) that

$$
\lim _{n \rightarrow \infty}\left\|T y_{n}-T p\right\|=0
$$


Journal of Inequalities and Applications

Since $P_{C}$ is firmly nonexpansive, we have

$$
\begin{aligned}
\left\|z_{n}-p\right\|^{2}= & \left\|P_{C}\left(I-t_{n} T\right) y_{n}-P_{C}\left(I-t_{n} T\right) p\right\|^{2} \\
\leq & \left\langle\left(I-t_{n} T\right) y_{n}-\left(I-t_{n} T\right) p, z_{n}-p\right\rangle \\
= & \frac{1}{2}\left(\left\|\left(I-t_{n} T\right) y_{n}-\left(I-t_{n} T\right) p\right\|^{2}+\left\|z_{n}-p\right\|^{2}\right. \\
& \left.\quad-\left\|\left(I-t_{n} T\right) y_{n}-\left(I-t_{n} T\right) p-\left(z_{n}-p\right)\right\|^{2}\right) \\
\leq & \frac{1}{2}\left(\left\|y_{n}-p\right\|^{2}+\left\|z_{n}-p\right\|^{2}-\left\|y_{n}-z_{n}-t_{n}\left(T y_{n}-T p\right)\right\|^{2}\right) \\
= & \frac{1}{2}\left(\left\|y_{n}-p\right\|^{2}+\left\|z_{n}-p\right\|^{2}-\left(\left\|y_{n}-z_{n}\right\|^{2}-2 t_{n}\left\langle y_{n}-z_{n}, T y_{n}-T p\right\rangle+t_{n}^{2}\left\|T y_{n}-T p\right\|^{2}\right)\right) .
\end{aligned}
$$

So, we obtain that

$$
\left\|z_{n}-p\right\|^{2} \leq\left\|x_{n}-p\right\|^{2}-\left\|y_{n}-z_{n}\right\|^{2}+2 t_{n}\left\|y_{n}-z_{n}\right\|\left\|T y_{n}-T p\right\| .
$$

It follows that

$$
\begin{aligned}
\left\|x_{n+1}-p\right\|^{2} & \leq \alpha_{n}\left\|x_{n}-p\right\|^{2}+\left(1-\alpha_{n}\right)\left\|S z_{n}-p\right\|^{2} \\
& \leq \alpha_{n}\left\|x_{n}-p\right\|^{2}+\left(1-\alpha_{n}\right)\left\|z_{n}-p\right\|^{2} \\
& \leq \alpha_{n}\left\|x_{n}-p\right\|^{2}+\left(1-\alpha_{n}\right)\left(\left\|x_{n}-p\right\|^{2}-\left\|y_{n}-z_{n}\right\|^{2}+2 t_{n}\left\|y_{n}-z_{n}\right\|\left\|T y_{n}-T p\right\|\right) .
\end{aligned}
$$

Therefore we have

$$
\left(1-\alpha_{n}\right)\left\|y_{n}-z_{n}\right\|^{2} \leq\left\|x_{n}-p\right\|^{2}-\left\|x_{n+1}-p\right\|^{2}+2 t_{n}\left\|y_{n}-z_{n}\right\|\left\|T y_{n}-T p\right\| .
$$

From the restriction (a) and (2.30), we get that

$$
\lim _{n \rightarrow \infty}\left\|y_{n}-z_{n}\right\|=0
$$

Note that

$$
\begin{aligned}
\left\|x_{n}-z_{n}\right\| & \leq\left\|x_{n}-y_{n}\right\|+\left\|y_{n}-z_{n}\right\| \\
& \leq \beta_{n}\left\|x_{n}-u_{n}\right\|+\left(1-\beta_{n}\right)\left\|x_{n}-v_{n}\right\|+\left\|y_{n}-z_{n}\right\| .
\end{aligned}
$$

From (2.17), (2.19), and (2.35), we obtain that

$$
\lim _{n \rightarrow \infty}\left\|x_{n}-z_{n}\right\|=0
$$


Define a mapping $R$ by

$$
R \mathrm{v}= \begin{cases}T v+N_{C} v, & v \in C, \\ \emptyset, & v \notin C .\end{cases}
$$

Let $(v, w) \in G(R)$. Since $w-T v \in N_{C} v$ and $z_{n} \in C$, we obtain that

$$
\left\langle v-z_{n}, w-T v\right\rangle \geq 0
$$

As $z_{n}=P_{C}\left(y_{n}-t_{n} T y_{n}\right)$ and $v \in C$, we get that

$$
\left\langle v-z_{n}, \frac{z_{n}-y_{n}}{t_{n}}+T y_{n}\right\rangle \geq 0
$$

From (2.39) and (2.40), we obtain that

$$
\begin{aligned}
\left\langle v-z_{n_{i}}, w\right\rangle & \geq\left\langle v-z_{n_{i}}, T v\right\rangle \\
& \geq\left\langle v-z_{n_{i}}, T v\right\rangle-\left\langle v-z_{n_{i}} \frac{z_{n_{i}}-y_{n_{i}}}{t_{n_{i}}}+T y_{n_{i}}\right\rangle \\
& =\left\langle v-z_{n_{i}}, T v-T y_{n_{i}}-\frac{z_{n_{i}}-y_{n_{i}}}{t_{n_{i}}}\right\rangle \\
& =\left\langle v-z_{n_{i}}, T v-T z_{n_{i}}\right\rangle+\left\langle v-z_{n_{i}}, T z_{n_{i}}-T y_{n_{i}}\right\rangle-\left\langle v-z_{n_{i}}, \frac{z_{n_{i}}-y_{n_{i}}}{t_{n_{i}}}\right\rangle \\
& \geq\left\langle v-z_{n_{i}}, T z_{n_{i}}-T y_{n_{i}}\right\rangle-\left\langle v-z_{n_{i}}, \frac{z_{n_{i}}-y_{n_{i}}}{t_{n_{i}}}\right\rangle .
\end{aligned}
$$

Note that $T$ is Lipschitz. On the other hand, we see from (2.37) that $z_{n_{i}} \rightarrow \bar{x}$. Hence, we get that

$$
\langle v-\bar{x}, w\rangle \geq 0
$$

Since $R$ is maximal monotone, we obtain that $\bar{x} \in R^{-1}(0)$. From Lemma 1.4, we get that $\bar{x} \in$ $V I(C, T)$.

Finally, we show that $\bar{x} \in F(S)$. Note that

$$
\begin{gathered}
\left\|x_{n+1}-p\right\| \leq \alpha_{n}\left\|x_{n}-p\right\|+\left(1-\alpha_{n}\right)\left\|S z_{n}-p\right\|, \\
\left\|S z_{n}-p\right\| \leq\left\|z_{n}-p\right\| \leq\left\|x_{n}-p\right\| .
\end{gathered}
$$


Since $\lim _{n \rightarrow \infty}\left\|x_{n}-p\right\|$ exists, we may assume that $\lim _{n \rightarrow \infty}\left\|x_{n}-p\right\|=r$ for some positive constant $r$. Then we see that

$$
\begin{gathered}
\underset{n \rightarrow \infty}{\limsup \left\|x_{n+1}-p\right\| \leq r, \quad \limsup _{n \rightarrow \infty}\left\|x_{n}-p\right\| \leq r,} \\
\limsup _{n \rightarrow \infty}\left\|S z_{n}-p\right\| \leq r .
\end{gathered}
$$

In view of Lemma 1.6, we get that

$$
\lim _{n \rightarrow \infty}\left\|x_{n}-S z_{n}\right\|=0
$$

Furthermore, we know that

$$
\begin{aligned}
\left\|S x_{n}-x_{n}\right\| & \leq\left\|S x_{n}-S z_{n}\right\|+\left\|S z_{n}-x_{n}\right\| \\
& \leq\left\|x_{n}-z_{n}\right\|+\left\|S z_{n}-x_{n}\right\| .
\end{aligned}
$$

From (2.37) and (2.45), we obtain that

$$
\lim _{n \rightarrow \infty}\left\|S x_{n}-x_{n}\right\|=0
$$

Note that $x_{n_{i}}-\bar{x}$ and $S x_{n_{i}}-x_{n_{i}} \rightarrow 0$ as $i \rightarrow \infty$. From Lemma 1.7, we arrive at $\bar{x} \in F(S)$. Assume that there exists another subsequence $\left\{x_{n_{j}}\right\}$ of $\left\{x_{n}\right\}$, converges to $x^{\prime}$, where $x^{\prime} \neq \bar{x}$. In view of the Opial's condition, we see that

$$
\begin{aligned}
\lim _{n \rightarrow \infty}\left\|x_{n}-\bar{x}\right\| & =\liminf _{i \rightarrow \infty}\left\|x_{n_{i}}-\bar{x}\right\|<\liminf _{i \rightarrow \infty}\left\|x_{n_{i}}-x^{\prime}\right\| \\
& =\lim _{n \rightarrow \infty}\left\|x_{n}-x^{\prime}\right\|=\liminf _{j \rightarrow \infty}\left\|x_{n_{j}}-x^{\prime}\right\| \\
& <\lim _{j \rightarrow \infty}\left\|x_{n_{j}}-\bar{x}\right\|=\lim _{n \rightarrow \infty}\left\|x_{n}-\bar{x}\right\| .
\end{aligned}
$$

This is a contradiction. So, we have $x^{\prime}=\bar{x}$.

Let $h_{n}=P_{\bar{q}} x_{n}$. Since $\bar{x} \in \mathcal{F}$, we have

$$
\left\langle x_{n}-h_{n}, h_{n}-\bar{x}\right\rangle \geq 0
$$

From (2.4) and Lemma 1.3, we get that $\left\{h_{n}\right\}$ converges strongly to some $v \in \mathcal{F}$. Since $\left\{x_{n}\right\}$ converges weakly to $\bar{x}$, we have

$$
\langle\bar{x}-v, v-\bar{x}\rangle \geq 0 .
$$


Hence we obtain that

$$
\bar{x}=v=\lim _{n \rightarrow \infty} P_{\bar{f}} x_{n} .
$$

This completes the proof.

Corollary 2.2. Let $C$ be a nonempty closed convex subset of a real Hilbert space $H$. Let $F$ be a bifunction from $C \times C$ to $\mathbb{R}$ which satisfies (A1)-(A4). Let $A: C \rightarrow H$ be an $\alpha$-inverse-strongly monotone mapping, $T: C \rightarrow H$ an $\lambda$-inverse-strongly monotone mapping, and $S: C \rightarrow C$ a nonexpansive mapping. Assume that $\mathcal{F}:=\mathrm{EP}(F, A) \cap V I(C, T) \cap F(S) \neq \emptyset$. Let $\left\{\alpha_{n}\right\}$ be a sequence in $[0,1]$. Let $\left\{a_{n}\right\}$ be a sequence in $[0,2 \alpha]$, and $\left\{t_{n}\right\}$ a sequence in $[0,2 \lambda]$. Let $\left\{x_{n}\right\}$ be a sequence generated in the following manner:

$$
\begin{gathered}
x_{1} \in C, \\
F\left(u_{n}, u\right)+\left\langle A x_{n}, u-u_{n}\right\rangle+\frac{1}{a_{n}}\left\langle u-u_{n}, u_{n}-x_{n}\right\rangle \geq 0, \quad \forall u \in C, \\
x_{n+1}=\alpha_{n} x_{n}+\left(1-\alpha_{n}\right) S P_{C}\left(u_{n}-t_{n} T u_{n}\right), \quad \forall n \geq 1 .
\end{gathered}
$$

Assume that the sequences $\left\{\alpha_{n}\right\},\left\{a_{n}\right\}$, and $\left\{t_{n}\right\}$ satisfy the following restrictions:

(a) $0<a^{\prime} \leq \alpha_{n} \leq a<1$;

(b) $0<d \leq a_{n} \leq e<2 \alpha, 0<h \leq t_{n} \leq j<2 \lambda$

for some $a^{\prime}, a, d, e, h, j \in \mathbb{R}$, then the sequence $\left\{x_{n}\right\}$ converges weakly to some point $\bar{x} \in \mathcal{F}$, where $\bar{x}=\lim _{n \rightarrow \infty} P_{\mp} x_{n}$.

Proof. Putting $F_{1}=F_{2}=F, A=B$, and $a_{n}=b_{n}$ in Theorem 2.1, we see that $y_{n}=u_{n}$. From the proof of Theorem 2.1, we can conclude the desired conclusion immediately.

Corollary 2.3. Let $C$ be a nonempty closed convex subset of a real Hilbert space $H$. Let $F$ be a bifunction from $C \times C$ to $\mathbb{R}$ which satisfies (A1)-(A4). Let $A: C \rightarrow H$ be an $\alpha$-inverse-strongly monotone mapping and $S: C \rightarrow C$ a nonexpansive mapping. Assume that $\mp:=\operatorname{EP}(F, A) \cap F(S) \neq \emptyset$. Let $\left\{\alpha_{n}\right\}$ be a sequence in $[0,1]$. Let $\left\{a_{n}\right\}$ be a sequence in $[0,2 \alpha]$. Let $\left\{x_{n}\right\}$ be a sequence generated in the following manner:

$$
\begin{gathered}
x_{1} \in C, \\
F\left(u_{n}, u\right)+\left\langle A x_{n}, u-u_{n}\right\rangle+\frac{1}{a_{n}}\left\langle u-u_{n}, u_{n}-x_{n}\right\rangle \geq 0, \quad \forall u \in C, \\
x_{n+1}=\alpha_{n} x_{n}+\left(1-\alpha_{n}\right) S u_{n}, \quad \forall n \geq 1 .
\end{gathered}
$$

Assume that the sequences $\left\{\alpha_{n}\right\}$ and $\left\{a_{n}\right\}$ satisfy the following restrictions:

(a) $0<a^{\prime} \leq \alpha_{n} \leq a<1$;

(b) $0<d \leq a_{n} \leq e<2 \alpha$ 
for some $a^{\prime}, a, d, e \in \mathbb{R}$, then the sequence $\left\{x_{n}\right\}$ converges weakly to some point $\bar{x} \in \mathcal{F}$, where $\bar{x}=$ $\lim _{n \rightarrow \infty} P_{\bar{\mp}} x_{n}$.

Proof. Putting $T=0$ in Corollary 2.2, we can conclude the desired conclusion immediately.

Remark 2.4. Corollary 2.3 is a generalization of Theorem 1.2 in Section 1. More precisely, Corollary 2.3 is reduced to Theorem 1.2 if $A=0$.

Corollary 2.5. Let $C$ be a nonempty closed convex subset of a real Hilbert space $H$. Let $A: C \rightarrow H$ be an $\alpha$-inverse-strongly monotone mapping, $B: C \rightarrow H$ a $\beta$-inverse-strongly monotone mapping, $T: C \rightarrow H$ an $\lambda$-inverse-strongly monotone mapping, and $S: C \rightarrow C$ a nonexpansive mapping. Assume that $\mathcal{F}:=\operatorname{VI}(C, A) \cap V I(C, B) \cap V I(C, T) \cap F(S) \neq \emptyset$. Let $\left\{\alpha_{n}\right\}$ and $\left\{\beta_{n}\right\}$ be sequences in $[0,1]$. Let $\left\{a_{n}\right\}$ be a sequence in $[0,2 \alpha],\left\{b_{n}\right\}$ a sequence in $[0,2 \beta]$, and $\left\{t_{n}\right\}$ a sequence in $[0,2 \lambda]$. Let $\left\{x_{n}\right\}$ be a sequence generated in the following manner:

$$
\begin{gathered}
x_{1} \in C, \\
y_{n}=\beta_{n} P_{C}\left(x_{n}-a_{n} A x_{n}\right)+\left(1-\beta_{n}\right) P_{C}\left(x_{n}-b_{n} B x_{n}\right), \\
x_{n+1}=\alpha_{n} x_{n}+\left(1-\alpha_{n}\right) S P_{C}\left(y_{n}-t_{n} T y_{n}\right), \quad \forall n \geq 1
\end{gathered}
$$

Assume that the sequences $\left\{\alpha_{n}\right\},\left\{\beta_{n}\right\},\left\{a_{n}\right\},\left\{b_{n}\right\}$, and $\left\{t_{n}\right\}$ satisfy the following restrictions:

(a) $0<a^{\prime} \leq \alpha_{n} \leq a<1,0<b \leq \beta_{n} \leq c<1$;

(b) $0<d \leq a_{n} \leq e<2 \alpha, 0<f \leq b_{n} \leq g<2 \beta, 0<h \leq t_{n} \leq j<2 \lambda$

for some $a^{\prime}, a, b, c, d, e, f, g, h, j \in \mathbb{R}$, then the sequence $\left\{x_{n}\right\}$ converges weakly to some point $\bar{x} \in \mathcal{F}$, where $\bar{x}=\lim _{n \rightarrow \infty} P_{\mp} x_{n}$.

Proof. Putting $F_{1}=F_{2}=0$, we see that

$$
\left\langle A x_{n}, u-u_{n}\right\rangle+\frac{1}{a_{n}}\left\langle u-u_{n}, u_{n}-x_{n}\right\rangle \geq 0
$$

is equivalent to

$$
u_{n}=P_{C}\left(x_{n}-a_{n} A x_{n}\right), \quad \forall n \geq 1
$$

In the same way, we can obtain that

$$
v_{n}=P_{C}\left(x_{n}-b_{n} B x_{n}\right), \quad \forall n \geq 1 .
$$

From the proof of Theorem 2.1, we can conclude the desired conclusion immediately.

Remark 2.6. Corollary 2.5 is a generalization of Theorem 1.1 in Section 1. More precisely, Corollary 2.5 is reduced to Theorem 1.1 if $T=0, A=B$, and $a_{n}=b_{n}$ for each $n \geq 1$. 


\section{Acknowledgments}

This work was supported by the Korea Research Foundation Grant funded by the Korean Government (KRF-2008-313-C00050) and the Science Research Foundation of Educational Department of Liaoning Province (2009A419).

\section{References}

[1] W. Takahashi and M. Toyoda, "Weak convergence theorems for nonexpansive mappings and monotone mappings," Journal of Optimization Theory and Applications, vol. 118, no. 2, pp. 417-428, 2003.

[2] A. Tada and W. Takahashi, "Weak and strong convergence theorems for a nonexpansive mapping and an equilibrium problem," Journal of Optimization Theory and Applications, vol. 133, no. 3, pp. 359-370, 2007.

[3] A. Moudafi, "On the convergence of splitting proximal methods for equilibrium problems in Hilbert spaces," Journal of Mathematical Analysis and Applications, vol. 359, no. 2, pp. 508-513, 2009.

[4] C. Jaiboon, P. Kumam, and U. W. Humphries, "Weak convergence theorem by an extragradient method for variational inequality, equilibrium and fixed point problems," Bulletin of the Malaysian Mathematical Sciences Society, vol. 32, no. 2, pp. 173-185, 2009.

[5] H. Iiduka and W. Takahashi, "Weak convergence theorems by Cesáro means for nonexpansive mappings and inverse-strongly-monotone mappings," Journal of Nonlinear and Convex Analysis, vol. 7, no. 1, pp. 105-113, 2006.

[6] H. Iiduka, W. Takahashi, and M. Toyoda, "Approximation of solutions of variational inequalities for monotone mappings," PanAmerican Mathematical Journal, vol. 14, no. 2, pp. 49-61, 2004.

[7] A. Moudafi, "Weak convergence theorems for nonexpansive mappings and equilibrium problems," Journal of Nonlinear and Convex Analysis, vol. 9, no. 1, pp. 37-43, 2008.

[8] W. Nilsrakoo and S. Saejung, "Weak and strong convergence theorems for countable Lipschitzian mappings and its applications," Nonlinear Analysis: Theory, Methods E Applications, vol. 69, no. 8, pp. 2695-2708, 2008.

[9] N. Nadezhkina and W. Takahashi, "Weak convergence theorem by an extragradient method for nonexpansive mappings and monotone mappings," Journal of Optimization Theory and Applications, vol. 128, no. 1, pp. 191-201, 2006.

[10] S. Plubtieng and P. Kumam, "Weak convergence theorem for monotone mappings and a countable family of nonexpansive mappings," Journal of Computational and Applied Mathematics, vol. 224, no. 2, pp. 614-621, 2009.

[11] J.-W. Peng and J.-C. Yao, "Weak convergence of an iterative scheme for generalized equilibrium problems," Bulletin of the Australian Mathematical Society, vol. 79, no. 3, pp. 437-453, 2009.

[12] X. Qin, Y. J. Cho, and S. M. Kang, "Convergence theorems of common elements for equilibrium problems and fixed point problems in Banach spaces," Journal of Computational and Applied Mathematics, vol. 225, no. 1, pp. 20-30, 2009.

[13] X. Qin, M. Shang, and Y. Su, "Strong convergence of a general iterative algorithm for equilibrium problems and variational inequality problems," Mathematical and Computer Modelling, vol. 48, no. 7-8, pp. 1033-1046, 2008.

[14] W. Takahashi and K. Zembayashi, "Strong and weak convergence theorems for equilibrium problems and relatively nonexpansive mappings in Banach spaces," Nonlinear Analysis: Theory, Methods $\mathcal{E}$ Applications, vol. 70, no. 1, pp. 45-57, 2009.

[15] R. T. Rockafellar, "On the maximality of sums of nonlinear monotone operators," Transactions of the American Mathematical Society, vol. 149, pp. 75-88, 1970.

[16] E. Blum and W. Oettli, "From optimization and variational inequalities to equilibrium problems," The Mathematics Student, vol. 63, no. 1-4, pp. 123-145, 1994.

[17] P. L. Combettes and S. A. Hirstoaga, "Equilibrium programming in Hilbert spaces," Journal of Nonlinear and Convex Analysis, vol. 6, no. 1, pp. 117-136, 2005.

[18] J. Schu, "Weak and strong convergence to fixed points of asymptotically nonexpansive mappings," Bulletin of the Australian Mathematical Society, vol. 43, no. 1, pp. 153-159, 1991.

[19] G. Marino and H.-K. Xu, "Weak and strong convergence theorems for strict pseudo-contractions in Hilbert spaces," Journal of Mathematical Analysis and Applications, vol. 329, no. 1, pp. 336-346, 2007. 